\title{
Formal Verification and Biology
}

\author{
David L. Dill \\ Department of Computer Science \\ Stanford University \\ dillastanford.edu
}

The essence of formal verification is the modeling, analysis, and, ultimately, understanding of large reactive systems. How do many parts interact to produce appropriate global behavior? How are properties guaranteed over all the possible variations of timing, non-deterministic behavior of components, and a dynamically changing environment?

Although formal verification has almost exclusively been applied to synthetic systems, I believe that these same types of questions will be of increasing interest in biology, and that similar techniques to those used in formal verification will be of value in answering them. The tutorial will discuss the rationale for modeling cellular processes as discrete transition systems and past work in modeling of biological systems using formalisms that parallel those in digital logic, along with state space search.

The conference talk will discuss two different applications of techniques from formal methods to modeling of cellular functions. The first is the use of Petri nets to model and analyze signal transduction pathways. Starting with a list of reactions in the cell, a tool was developed that can interactively answer user queries about possible cell states by solving a Petri net reachability problem in real-time, then displaying a diagram of the reactions that lead to the result.

The second application is the use of symbolic model checking to analyze the steps of the cell cycle in the bacterial cell Caulobacter Crescentus. The cell cycle even in simple organism is essentially a complex asynchronous circuit that must function reliably despite the presence of substantial noise, environmental perturbations, and variations in reaction rates due to other causes. Our findings were that, surprisingly, the cell cycle is almost completely robust to arbitrary variations in the timing of cell cycle events. 